\title{
Use of prototype automated blood culture system and gas-liquid chromatography for the analysis of continuous ambulatory peritoneal dialysis associated infection
}

\author{
C R Catchpole, F Macrae, J D Brown, M Palmer, D E Healing, N T Richards, \\ T S J Elliott
}

\begin{abstract}
Aims-(1) To compare the recovery of organisms from continuous ambulatory peritoneal dialysis (CAPD) effluent fluid obtained from patients with clinical evidence of peritonitis, with an automated system (AS) and the Septichek blood culture system; (2) to evaluate the times to detection of organisms with the two systems; (3) to identify anaerobes from CAPD samples by extended anaerobic culture and gas-liquid chromatography (GLC).

Methods-168 CAPD effluent fluid samples were studied, representing 157 episodes of peritonitis in 97 patients. CAPD samples were inoculated into two AS bottles-one anaerobic, one aerobic-and a Septichek bottle; samples were also examined for cell count, Gram stain, and direct culture. Culture bottles were then subcultured onto various media, and any organisms isolated were identified. After routine culture, GLC was performed on culture fluid in the anaerobic AS and Septichek bottles. When volatile fatty acids were detected, the broths were cultured anaerobically on specialised medium for a further five days.
\end{abstract}

Results-147 organisms were isolated from the 168 samples: $96(57 \%)$ yielded growth of significant organisms by direct culture, as compared to $129(76.8 \%)$ by both AS and Septichek. There was no significant difference in isolation rates between AS and Septichek, but time to detection was more rapid with the AS system $(p<0.002)$. GLC showed volatile fatty acid in 15 specimens; of these, 14 subsequently grew anaerobic organisms.

Conclusions-AS was comparable to Septichek for numbers of isolations. Speed to detection was faster with the AS, which may be an advantage in management of patients with CAPD peritonitis. GLC showed anaerobes in several cases which would not have been detected without prolonged anaerobic culture; thus anaerobic cultures are recommended for patients who are unresponsive to antimicrobials or who have evidence of bowel perforation. (F Clin Pathol 1997;50:241-244)
Keywords: culture systems; continuous ambulatory peritoneal dialysis; anaerobes.

Continuous ambulatory peritoneal dialysis (CAPD) is now an established treatment for patients with end stage renal failure. However, peritonitis remains a major complication, not only associated with a recognised morbidity and mortality, but also with increased costs. Conventional laboratory diagnosis of CAPD infection includes a white blood cell count, Gram stain, and culture of dialysis fluid. ${ }^{1}$ Gram stains of centrifuged deposits and direct culture of CAPD effluent fluid are relatively insensitive methods for detecting organisms. ${ }^{1-5}$ Enrichment techniques, including the inoculation of dialysis fluid into blood culture bottles, have been shown to yield a higher percentage of positive cultures. $^{245}$ However, there remain significant numbers of patients with clinical peritonitis in whom conventional cultures fail to reveal a pathogen.

The treatment of suspected CAPD associated peritonitis is often initiated before the results of cultures are available, and empirical treatment usually comprises broad spectrum antimicrobial agents. As initial Gram staining of effluent fluid is generally an insensitive method for the identification of the infecting organism, rapid detection of positive cultures may prove to be of benefit in reducing the unnecessary use of expensive and potentially toxic drugs, and allow directed treatment to be started earlier.

In this study, a novel automated blood culture system (Unipath Ltd, Basingstoke, United Kingdom), in which recognition of positive cultures is based on the detection of changes in the headspace pressure in culture bottles by means of high resolution laser scanning in a flexible sealing septum, ${ }^{6}$ was used for the culture of CAPD effluent fluid. Preliminary laboratory and clinical trials have shown that the prototype system is capable of supporting the growth of a wide range of organisms in blood cultures, and provides rapid detection of positive cultures compared to other systems. ${ }^{6} \mathrm{~A}$ preliminary clinical evaluation of a new aerobic blood culture medium containing saponin for use with this automated system (AS) enhanced further the time to detection rate of staphylococci, Escherichia coli, and yeasts from blood cultures. 
The aims of the study were to compare the recovery of organisms from CAPD effluent fluid obtained from patients with clinical evidence of peritonitis using the AS and the Septichek blood culture system (Becton Dickinson Microbiology Systems, Cockeysville, Maryland, USA) which has been shown to provide enhanced recovery of organisms over direct plating methods. ${ }^{2}$ The time to detection of organisms from CAPD fluid with the AS system was also compared with the manual culture system.

The presence of anaerobic organisms in CAPD effluent fluid has been reported infrequently, ${ }^{18} 9$ and detection has been associated with bowel perforation. ${ }^{1}$ In this study the presence of anaerobes in CAPD effluent fluid was also investigated by inoculation of dialysate into AS blood culture bottles formulated to improve the recovery of anaerobic organisms, and by performing gas-liquid chromatography (GLC) on the blood culture bottle broth at the end of the routine culture period, with further prolonged anaerobic subculture of those in which volatile fatty acids were detected.

\section{Methods}

PATIENTS

Adult patients attending the CAPD clinic at the Queen Elizabeth Hospital, Birmingham, United Kingdom, who underwent CAPD effluent fluid analysis for suspected peritonitis were included in the study. Patients are routinely advised to attend the clinic if the dialysis fluid becomes cloudy or if they feel generally unwell with fever or abdominal pain, and if blockage of the CAPD catheter or signs of an exit site infection develop. A presumptive diagnosis of peritonitis is made when the effluent fluid from a patient with appropriate symptoms or signs is confirmed as being due to a raised white blood cell count (WBC $>100 / \mathrm{mm}^{3}$ ). Broad spectrum antimicrobials are started on an empirical basis, or selection guided by the initial Gram stain of dialysis fluid. The clinical symptoms or signs of

Table 1 Organisms isolated from 168 samples representing 157 episodes of peritonitis

\begin{tabular}{|c|c|c|c|c|c|}
\hline Organism & Direct culture & AS only & Septichek only & Both systems & Total \\
\hline $\begin{array}{l}\text { Coagulase-negative } \\
\text { staphylococci }\end{array}$ & 60 & 5 & 4 & 63 & 72 \\
\hline Staphylococcus aureaus & 8 & 0 & 1 & 9 & 10 \\
\hline Micrococcus species & 1 & 0 & 1 & 3 & 4 \\
\hline Enterococcus faecalis & 0 & 0 & 0 & 2 & 2 \\
\hline Enterococcus faecium & 1 & 2 & 0 & 3 & 5 \\
\hline Streptococcus mitis & 1 & 0 & 0 & 6 & 6 \\
\hline Streptococcus sanguis & 1 & 0 & 0 & 4 & 4 \\
\hline Streptococcus salivarius & 2 & 0 & 0 & 3 & 3 \\
\hline Streptococcus agalactiae & 1 & 0 & 0 & 1 & 1 \\
\hline Diphtheroid organism & 5 & 1 & 1 & 6 & 8 \\
\hline Bacillus species & 0 & 0 & 0 & 2 & 2 \\
\hline Escherichia coli & 2 & 0 & 0 & 3 & 3 \\
\hline Klebsiella species & 5 & 0 & 0 & 4 & 4 \\
\hline Acinetobacter species & 3 & 0 & 0 & 6 & 6 \\
\hline Citrobacter species & 1 & 0 & 0 & 1 & 1 \\
\hline Enterobacter species & 1 & 0 & 0 & 1 & 1 \\
\hline Serratia marcescens & 1 & 0 & 1 & 1 & 2 \\
\hline Pseudomonas chlorescens & 1 & 0 & 1 & 0 & 1 \\
\hline Stenotrophomonas maltophilia & 1 & 0 & 0 & 1 & 1 \\
\hline Bacteroides species & 0 & 0 & 0 & 3 & 3 \\
\hline Veillonella species & 0 & 0 & 0 & 2 & 2 \\
\hline Mixed anaerobes & 0 & 2 & 0 & 0 & 2 \\
\hline Candida species & 4 & 0 & 0 & 4 & 4 \\
\hline Total & 99 & 10 & 9 & 128 & 147 \\
\hline
\end{tabular}

Total number of positive samples $=138$.

AS = automated system. patients entering the study were recorded, together with details of any antimicrobial treatment given before and after collection of specimens for analysis.

\section{LABORATORY PROCEDURES}

Samples were drawn from the dialysis fluid bags aseptically using a sterile needle and syringe. Each of the two AS bottles (one aerobic bottle containing saponin and one anaerobic bottle) and the Septichek bottle were inoculated with $10 \mathrm{ml}$ of dialysate, and a further $10 \mathrm{ml}$ were inoculated into a sterile universal container for a cell count, Gram stain, and direct culture. A cell count was performed with a modified Fuchs Rosenthal chamber. The remaining fluid in the universal container was centrifuged for 10 minutes at $3000 \mathrm{rpm}$, and the deposit used to perform a Gram stain and direct culture. Two blood agar plates (one containing Tween 80) and a CLED plate (Oxoid, United Kingdom) were inoculated with the centrifuged deposit and incubated in air supplemented with $5 \% \mathrm{CO}_{2}$ at $37^{\circ} \mathrm{C}$ for 48 hours.

The AS bottles were loaded into the automated machine. Bottle headspace pressure was monitored every five minutes and a positive detection automatically flagged. A paddle device was attached to each Septicheck bottle according to the manufacturer's instructions, and cultures incubated in air at $37^{\circ} \mathrm{C}$. All bottles were inverted daily (to inoculate the agar paddle) and visually monitored at 0900 and at regular intervals during the day according to usual laboratory practice. A bottle was considered positive if the broth was turbid or organisms were observed growing on the agar of the paddle device. All bottle cultures were maintained until a positive growth was detected, or, if negative, for seven days. Terminal subculture was performed from all culture broths. A Gram stain was performed on positive cultures and the broth subcultured onto appropriate media and incubated in aerobic and anaerobic atmospheres for 48 hours. All organisms were identified according to routine laboratory practice, and appropriate antimicrobial susceptibility tests performed.

At the end of the routine culture period (seven days), AS anaerobic and Septichek bottles were sampled and GLC performed on the culture fluid to detect the presence of volatile fatty acids. If volatile fatty acids were detected, the broth was subcultured onto three $10 \%$ blood agar plates (one without supplements, one with nalidixic acid and Tween 80 , and one with added haemin and menandione, nalidixic acid, and vancomycin) (Oxoid), and cultured anaerobically for a further five days undisturbed in the anaerobic cabinet. Any organisms isolated were identified according to routine laboratory practice.

\section{Results}

During the study period, 223 specimens were processed in the laboratory. Fifty five specimens were excluded from the analysis as the patients had no clinical evidence of peritonitis and the WBC of the effluent fluid specimen 
Table 2 Times to detection of positive cultures, from receipt in the laboratory, by the AS and Septichek culture systems

\begin{tabular}{llll}
\hline & \multicolumn{2}{l}{ Mean detection time (hours) } \\
\cline { 2 - 3 } Organism isolated & $A S$ & Septichek & \multirow{2}{*}{$p$} \\
\hline Coagulase negative staphylococci $(\mathrm{n}=44)$ & 21 & 33.7 & $<0.002$ \\
All Gram positive aerobes $(\mathrm{n}=66)$ & 18.7 & 36.5 & $<0.002$ \\
All Gram negative aerobes $(\mathrm{n}=5)$ & 11.8 & 39.3 & $\star$ \\
Yeasts $(\mathrm{n}=1)$ & 10.7 & 42 & $\star$ \\
All organisms including polymicrobial cultures $(\mathrm{n}=78)$ & 17.4 & 37.4 & $<0.002$ \\
\hline
\end{tabular}

^Statistical analysis not performed.

was less than $100 / \mathrm{mm}^{3}$. The remaining 168 samples represented 157 discrete episodes of peritonitis in 97 patients. The organisms isolated by direct culture, AS, and Septichek are shown in table 1 . There were 147 organisms isolated. Ninety six samples (57\%) yielded growth of significant organisms by direct culture, as compared with $129(76.8 \%)$ by AS and $129(76.8 \%)$ by Septichek. Overall, 138 samples ( $82 \%$ ) were culture positive by one or more method, representing 135 episodes of peritonitis with positive cultures $(86 \%) ; 128$ of the 147 organisms were recovered by both the broth culture methods. Ten samples yielded organisms in the AS (five Staphylococcus epidermidis, two Enterococcus spp, one diphtheroid organism, and two yielding mixed anaerobic organisms) which were not recovered by Septichek; whereas nine organisms (four Staphylococcus epidermidis, one Staphylococcus aureus, one Micrococcus sp, one diphtheroid organism, one Serratia marsescens, and one Pseudomonas chlorescens) were not isolated from the corresponding AS bottles. All culture methods yielded the four Candida spp recovered during the study. Forty nine per cent of all organisms isolated were coagulase negative staphylococci and $12.9 \%$ of isolates were Gram negative aerobic organisms.

Organisms were seen in the initial Gram stained smear of the centrifuged deposit in 12 of the 138 positive samples $(8.7 \%)$. Data were available on the time to detection from receipt in the laboratory of 78 positive samples where organisms were isolated in both systems, and are shown in table 2. Overall, the time to detection of organisms in the AS was faster than for Septichek for all organisms recovered $(\mathrm{p}<0.002)$.
Table 4 Indication for culture of CAPD fluid

\begin{tabular}{lcc}
\hline $\begin{array}{l}\text { Indication for collection } \\
\text { of fluid }\end{array}$ & $\begin{array}{l}\text { Number of } \\
\text { samples }\end{array}$ & $\begin{array}{l}\text { Number culture } \\
\text { positive }\end{array}$ \\
\hline Cloudy fluid & 138 & 116 \\
Abdominal pain & 93 & 82 \\
Fever & 36 & 30 \\
Exit site infection & 7 & 7 \\
Nausea/vomiting & 5 & 4 \\
\hline
\end{tabular}

${ }^{\star}$ Many patients had more than one indication.

Further analysis of 141 patient samples was performed by GLC on the AS anaerobic and Septichek broths at the end of the routine culture period. In 15 specimens, volatile fatty acids were detected and on prolonged anaerobic culture (for five days) 14 of these specimens grew anaerobic organisms (table 3 ). One sample gave a volatile fatty acid profile typical of Bacteroides spp; however, extended anaerobic culture failed to recover any organisms. By comparison, only five of these patient samples grew anaerobic organisms during the routine culture period.

Cloudy fluid was the most often recorded indication for CAPD effluent fluid culture (table 4), followed by abdominal pain, which was more common in those patients with culture positive fluid. Fever was recorded in only a minority of cases.

Of the 10 samples taken from patients recorded as receiving antimicrobials at the time of sampling, five yielded growth on conventional culture. One sample contained Candida spp, two had Staphylococcus epidermidis, another had Enterococcus faecium (resistant to vancomycin), Veillonella spp, and Bacteroides fragilis, and the final sample grew Acinetobacter spp. The organisms isolated were resistant to the current antimicrobial therapy in four cases.

\section{Discussion}

Inoculation of CAPD fluid into blood culture bottles has become a routine method used in the investigation of CAPD peritonitis, and has been recommended by a working party of the British Society for Antimicrobial Chemotherapy (1987) to increase the yield of positive cultures. ${ }^{1}$ Other methods of increasing the detection of organisms include lysis centrifugation, filtration, and the use of pour plates. All

Table 3 Isolation of anaerobes from CAPD fluid samples

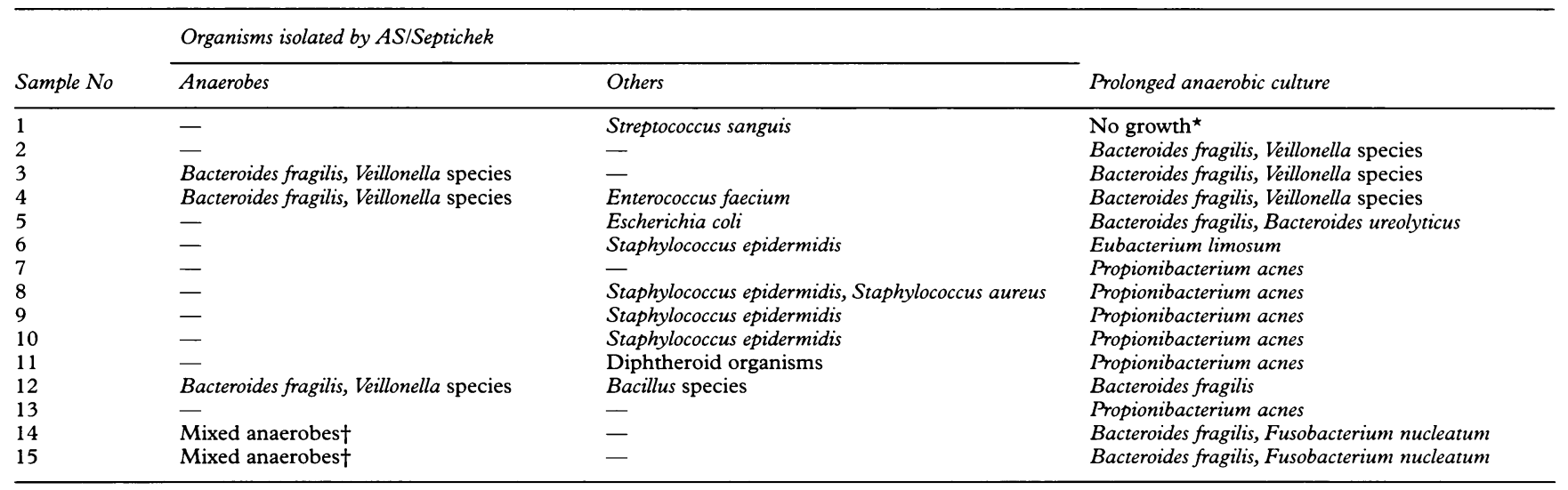

Volatile fatty acids were detected in all samples.

* The GLC profile was suggestive of Bacteroides fragilis.

$\dagger$ Individual identification of organisms not performed. 
these methods offer improved detection rates compared with direct culture and produce a quantitative result. ${ }^{510-14}$ However, they are more technically demanding than simple broth enrichment.

The isolation rates of organisms from CAPD effluent fluid should be assessed in the context of the diagnostic criteria used to define peritonitis. In this study, peritonitis was considered to be present if clinical symptoms and signs of infection were associated with CAPD effluent fluid containing more than $100 \mathrm{WBC} / \mathrm{mm}^{3}$ of fluid. Other workers have used a higher cut off to define a significant WBC count, ${ }^{5}$ which, if applied to this study, would have resulted in an apparently greater proportion of episodes of infection associated with a positive culture. However, the rate for positive culture in clinical peritonitis in this study of $86 \%$ is within the BSAC Working Party recommendations. ${ }^{1}$

The aim of this present study was to assess the use of a prototype automated blood culture system for the diagnosis of CAPD peritonitis. Our results showed that the automated system was able to support the growth of organisms from CAPD effluent fluid, and that isolation rates were comparable to the established manual broth system used as the control (Septichek). The results also confirmed the relatively poor performance of direct culture for the diagnosis of CAPD infection. Time to detection of positive cultures was, however, significantly faster with the automated system than with the manual system. The rapid detection of positive cultures may allow antimicrobial treatment to be rationalised and ensure appropriate therapy is initiated earlier, thus avoiding potential toxicity and reducing the likelihood of antimicrobial resistance emerging. Similarly, reduction in costs of patient management, ranging from antimicrobial agents to hotel charges, could be achieved.

The relatively high number of effluent fluid samples yielding anaerobic organisms after more detailed investigation has not been reported before..$^{7-9}$ This may reflect the more fastidious approach to the detection of anaerobes used in this study, and the prolonged anaerobic subculture at the end of the "routine" culture period. Of note was the isolation of Propionibacterium acnes from six broth cultures after prolonged anaerobic incubation (table 3). Three of these isolates were from different specimens taken on separate days from the same patient. This repeatable positivity suggests that the isolates which are associated with skin colonisation were unlikely to be contaminants. In a further four CAPD samples, anaerobic skin commensals were also isolated. These results suggest that the skin rather than the gastrointestinal tract was the source of the anaerobic infection in these cases. The other anaerobic organisms isolated in the study are associated with gastrointestinal colonisation, and this seems likely to have been the source of infection.

GLC is clearly not practicable for analysis of every CAPD effluent specimen taken. However, in patients who fail to respond to apparently adequate treatment, or those in whom anaerobic infection originating from perforation of a viscus is suspected, further investigation of apparently culture negative samples by prolonged anaerobic culture may be warranted.

1 Bint AJ, Finch RG, Gokal R, Goldsmith B, Junor B, Oliver $D$. Diagnosis and management of peritonitis in continuous ambulatory peritoneal dialysis: report of a working party of the British Society for Antimicrobial Chemotherapy. Lancet 1987; i:845-8.

2 Ryan S, Fessia S. Improved method for recovery of peritonitis causing microorganisms from peritoneal dialysate. $\mathcal{F}$ Clin tis causing microorganisms

3 Knight KR, Polak A, Crump J, Maskell R. Laboratory diagnosis and oral treatment of CAPD peritonitis. Lancet 1982; ii: $1301-4$

4 Doyle P, Chrichton E, Mathias R, Weib R. Clinical and microbiological evaluation of four culture methods for the diagnosis of peritonitis in patients on continuous ambulatory peritoneal dialysis. 7 Clin Microbiol 1989;27:1206-9.

5 Males BM, Walshe JJ, Garringer L, Koscinski D, Amersterdam D. Addi-chek filtration, BACTEC, and $10 \mathrm{ml}$ culture methods for recovery of micro-organisms from dialysis effluent during episodes of peritonitis. $\mathcal{f}$ Clin Microbiol 1986;23:350-3.

6 Stevens CM, Swaine D, Butler C, Carr AH, Weightman A, Catchpole CR, et al. Development of o.a.s.i.s., a new automated blood culture system in which detection is based on measurement of bottle headspace pressure changes. $\mathcal{f}$ Clin Microbiol 1994;32:1750-6.

7 Elliott TSJ, Catchpole CR, Healing DE, Palmer MD, Hart IT. Evaluation of a new microbial rescue medium in a novel blood culture system. Abstract, 93rd General Meeting of blood culture system. Abstract, 93rd General Meeting
American Society of Microbiology, Las Vegas 1994.

8 Vas SI. Infections associated with peritoneal and haemodialysis. In: Bisno AL, Waldvogel FA, eds. Infections associated with indwelling medical devices. Washington DC: American Society for Microbiology 1989:215-48.

9 Peterson PK, Matzke G, Keane WF. Current concepts in the management of peritonitis in patients undergoing conthe management of peritonitis in patients undergoing con-
tinuous ambulatory peritoneal dialysis. Rev Infect Dis 1987; 9:604-12.

10 Ludlam H, Dickens A, Simpson A, Phillips I. A comparison of four culture methods for diagnosing infection in continuous ambulatory peritoneal dialysis. $\mathcal{F}$ Hosp Infect 1990;16:263-9.

11 Ludlam HA, Price TNC, Berry AJ, Phillips I. Laboratory diagnosis of peritonitis in patients on continuous ambulatory peritoneal dialysis. 7 Clin Microbiol 1988;26:1757-62.

12 Fenton P. Laboratory diagnosis of peritonitis in patients undergoing continuous ambulatory peritoneal dialysis. $f$ Clin Pathol 1982;35:1181-4.

13 Spencer RC, Ahmed WK. Laboratory diagnosis of peritonitis in continuous peritoneal dialysis by lysis and centrifugatis in continuous peritoneal dialysis
tion. Technical Methods 1986:925-6.

14 Taylor PC. Routine laboratory diagnosis of continuous ambulatory peritoneal dialysis using centrifugation/lysis and saponin-containing media. Eur $\mathcal{F}$ Clin Microbiol Infect Dis 1994;13:249-52. 